\title{
Choroid Plexitis as a Unique Neurological Manifestation in Granulomatosis with Polyangiitis (Wegener's Disease)
}

SALVADOR SIERRA, MD, PhD, NATASHA LUQUIN, PhD, Division of Neurosciences, Center for Applied Medical Research, University of Navarra, Pamplona; MANUEL TARDÁGUILA, MD, Department of Neurosurgery, Hospital Universitari Germans Trias i Pujol, Badalona; ALEJANDRO OLIVÉ, MD, PhD, Department of Rheumatology, Hospital Universitari Germans Trias i Pujol, Badalona, Spain. Address correspondence to Dr. Sierra, Neurosciences Division Center for Applied Medical Research, University of Navarra, Pio XII Avenue 55, 31008 Pamplona, Spain; E-mail: ssierra@alumni.unav.es. J Rheumatol 2014;41:1192-3; doi:10.3899/jrheum.131302

A 70-year-old man presented with a 3-day history of nausea, vomiting and constitutional symptoms (fatigue and anorexia). During hospitalization he developed diplopia. Cranial magnetic resonance imaging with gadolinium (Figure 1a) showed inflammation of the entire choroid plexus. A diagnosis of granulomatosis with polyangiitis (GPA) was made based on: presence of perinuclear antineutrophil cytoplasmic antibody at titer $1 / 40$ with elevated antiproteinase 3 antibodies; sinus radiography showing a mucosal thickening of the left maxillary sinus; and a lung biopsy demonstrating chronic granulomatous inflammation with multinucleated giant cells. The patient was treated with methylprednisolone [ $1 \mathrm{~g} /$ day intravenously (IV) for 3 days], cyclophosphamide $\left(0.6 \mathrm{mg} / \mathrm{m}^{2} /\right.$ dose IV, monthly pulses for 6 mos) and mycophenolate mofetil (2 g/day PO, maintenance therapy). He made a full recovery. A followup MRI showed partial improvement of the inflammation (Figure 1B).

Although not commonly the initial symptom ${ }^{1,2}$, the most frequent pattern of central nervous system involvement is
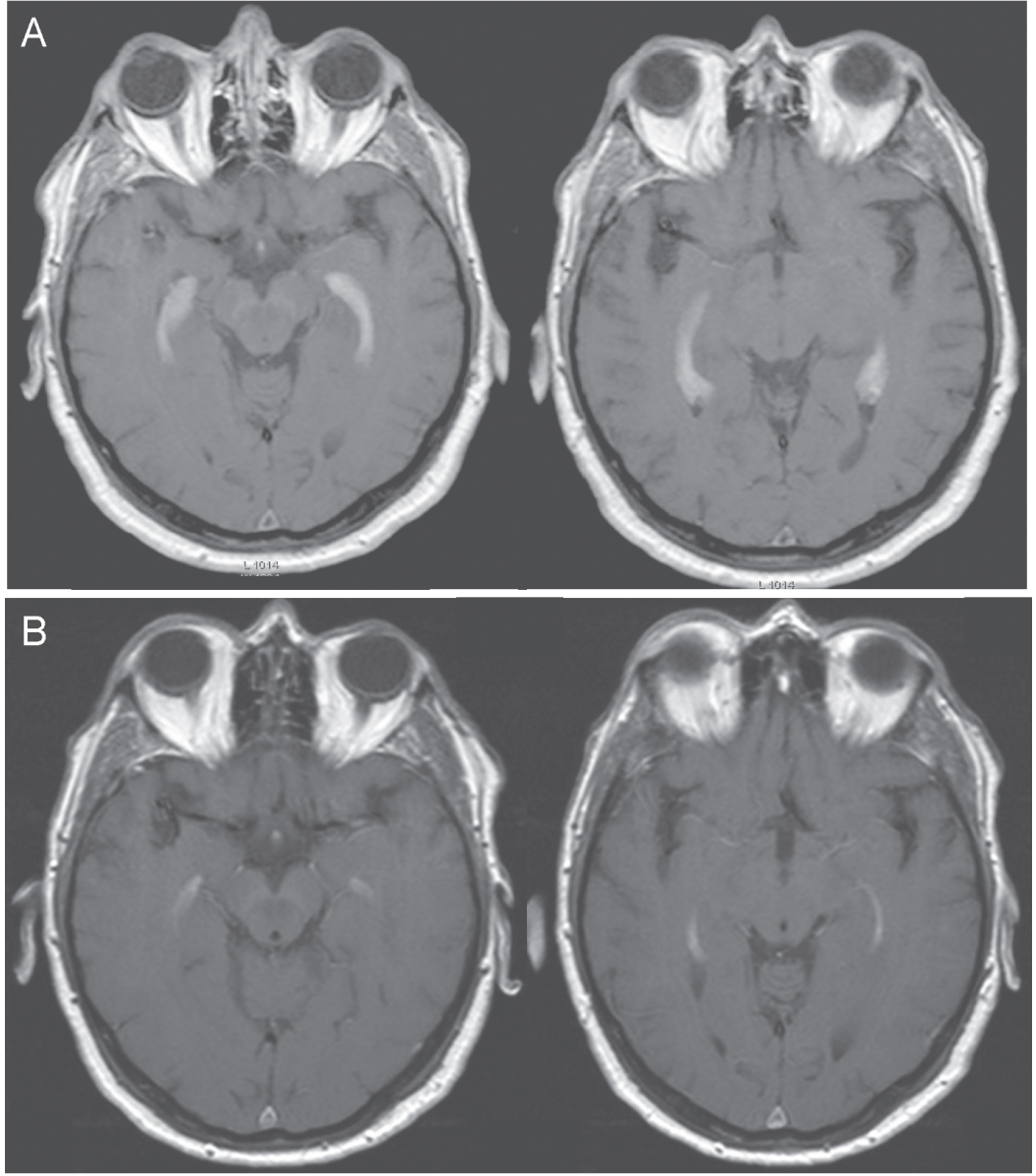

Figure 1. Axial contrast-enhanced T1-weighted images. Homogenous marked enhancement of enlarged choroid plexus within the 2 temporal horns (a); and followup imaging 3 months after immunosuppression therapy showing a prominent reduction in size and enhancement (b). 
granulomatous inflammation that leads to compression of usually cranial nerves II-III, V-VIII, meningitis and pituitary gland inflammation ${ }^{3}$. Our literature review of choroid plexus involvement in GPA cases yielded no reports.

\section{ACKNOWLEDGMENT}

The authors thank Mireia Tomás for assistance in acquiring patient data for this report.

\section{REFERENCES}

1. Nishino H, Rubino FA, Parisi JE. The spectrum of neurologic involvement in Wegener's granulomatosis. Neurology 1993;43:1334-7.

2. de Groot K, Schmidt DK, Arlt AC, et al. Standardized neurologic evaluations of 128 patients with Wegener granulomatosis. Arch Neurol 2001;58:1215-21.

3. Holle JU, Gross WL. Neurological involvement in Wegener's granulomatosis. Curr Opin Rheumatol 2011;23:7-11. 